\title{
Control of PDMS crosslinking by encapsulating a hydride crosslinker in a PMMA
} microcapsule

Ma, Baoguang; Hansen, Jens Henrik; Hvilsted, Søren; Skov, Anne Ladegaard

Published in:

R S C Advances

Link to article, DOI:

$10.1039 / \mathrm{c} 4 \mathrm{ra} 07513 \mathrm{~g}$

Publication date:

2014

Document Version

Publisher's PDF, also known as Version of record

Link back to DTU Orbit

Citation (APA):

Ma, B., Hansen, J. H., Hvilsted, S., \& Skov, A. L. (2014). Control of PDMS crosslinking by encapsulating a hydride crosslinker in a PMMA microcapsule. R S C Advances, 4(88), 47505-47512.

https://doi.org/10.1039/c4ra07513g

\section{General rights}

Copyright and moral rights for the publications made accessible in the public portal are retained by the authors and/or other copyright owners and it is a condition of accessing publications that users recognise and abide by the legal requirements associated with these rights.

- Users may download and print one copy of any publication from the public portal for the purpose of private study or research.

- You may not further distribute the material or use it for any profit-making activity or commercial gain

- You may freely distribute the URL identifying the publication in the public portal 


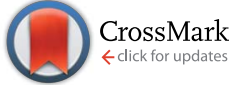

Cite this: RSC Adv., 2014, 4, 47505
Received 23rd July 2014

Accepted 19th September 2014

DOI: $10.1039 / \mathrm{c} 4 \mathrm{ra0} 7513 \mathrm{~g}$

www.rsc.org/advances

\section{Control of PDMS crosslinking by encapsulating a hydride crosslinker in a PMMA microcapsule $\uparrow$}

\begin{abstract}
Baoguang Ma, Jens Henrik Hansen, ${ }^{b}$ Søren Hvilsted ${ }^{a}$ and Anne Ladegaard Skov*a
In the preparation of PDMS elastomers, a combination of mixing and reactive processes constrains the applicability of the PDMS elastomer in research and applications. Separation of the mixing and reactive processes, which control PDMS crosslinking, has been achieved by encapsulating a hydride crosslinker in a PMMA shell. Microcapsules are mixed with vinyl-terminated PDMS to create a gelation system, which allows for storage at $50{ }^{\circ} \mathrm{C}$, without premature gelation, and in addition allows for extensive crosslinking reaction at $120^{\circ} \mathrm{C}$. Both visual observations and rheological studies show that a robust PDMS elastomer is obtained upon heating the gelation system. Furthermore, the influence of stoichiometric imbalance on the equilibrium storage modulus of the PDMS network is investigated, by employing different amounts of microcapsules in vinyl-terminated PDMS. It has been found that adding microcapsules increases the equilibrium storage modulus of the PDMS elastomer until the diffusion of the hydride crosslinker is constricted. An optimum amount of crosslinker used in the control crosslinking reaction has also been found. However, compared to the pure PDMS elastomer, the modulus of the PDMS elastomer from the encapsulated system is less sensitive in relation to the stoichiometry of the system than the corresponding polymer network. This broadens the applicability range of silicone elastomers.
\end{abstract}

\section{Introduction}

A controlled crosslinking reaction, which takes place in response to external stimulus, ${ }^{1}$ is of considerable interest in applications. Amongst all research on and applications of silicone elastomers, controlling the crosslinking reaction (e.g. between a vinyl-terminated PDMS and a hydride crosslinker) attracts most attention, due to the fascinating properties of PDMS elastomers obtained from the reaction. ${ }^{2}$ One potential application where control of PDMS crosslinking reaction is required is in the delivery of elastic seals to fractures in oil reservoirs. In the sealing process, the crosslinking reaction needs to be postponed until the elastomer reactants are placed inside the fracture. One way to solve the problem is to encapsulate the crosslinker in polymeric microcapsules. ${ }^{3}$ When the hydride crosslinker is sequestered inside the polymeric microcapsules, the microcapsules can be mixed with the vinylterminated PDMS, without undergoing a crosslinking reaction, thereby ensuring the mixture has not reacted. ${ }^{4}$

In order to release the hydride crosslinker from the polymeric shell, the shell of the microcapsule should be capable of

${ }^{a}$ Danish Polymer Centre, Department of Chemical and Biochemical Engineering, Technical University of Denmark, Søltofts Plads Building 227, DK-2800 Kgs. Lyngby, Denmark.E-mail: al@kt.dtu.dk

${ }^{b}$ Maersk Oil Research and Technology Centre, Education City, P.O. Box 210112, Doha, Qatar

$\dagger$ Electronic supplementary information (ESI) available. See DOI: 10.1039/c4ra07513g changing its morphology or structure upon external stimulus. ${ }^{5}$ Thermal initiation is one of the most commonly used stimuli which causes changes in the polymer structure of amorphous polymer. $^{6}$ At temperatures lower than the glass transition temperature $\left(T_{\mathrm{g}}\right)$ of the polymeric shell, the polymeric shell remains rigid and the hydride crosslinker is sequestered inside it; therefore, the mixture containing the microcapsules and vinyl-terminated PDMS will remain liquid-like, due to the absence of a crosslinking reaction. Upon heating up the mixture, ${ }^{7}$ the structure of the polymeric shell changes when the temperature is higher than its $T_{\mathrm{g}}$, resulting in the release of the hydride crosslinker and the initiation of the crosslinking reaction.

Traditionally so-called model networks are prepared from silicone networks obtained by silylation reactions. ${ }^{8-10}$ A (usually short) hydride functional crosslinker is reacted with long, linear, end-linked vinyl functional silicones to yield a network. The silylation reaction is catalyzed by platinum. During the crosslinking reaction, the vinyl-terminated PDMS and the hydride crosslinker convert into one large, infinite molecule. ${ }^{\mathbf{1 1}}$ Consequently, the mixture loses its solubility, and its storage modulus starts to rise to a finite value until the completion of the crosslinking reaction. ${ }^{12}$ The storage modulus at the completion of the crosslinking reaction refers to the equilibrium storage modulus, which is determined by stoichiometric imbalance and the crosslinking density of the network. Many studies show that the equilibrium storage modulus of the PDMS network is sensitive to the stoichiometric imbalance as well as 
the perfection of the PDMS network. ${ }^{\mathbf{1 1 , 1 3 - 1 6}}$ Thus, the influence of stoichiometric imbalance on the equilibrium storage modulus will be investigated, in order to determine the optimum amount of microcapsules and sensitivity of the storage modulus on stoichiometric imbalance in controlled PDMS crosslinking reaction.

This paper, which is devoted to studying the crosslinking reaction between a hydride crosslinker and a vinyl-terminated PDMS polymer controlled by releasing the hydride crosslinker from a PMMA shell, is divided into the following parts: in the first part, we describe the preparation and the characterisation of a PMMA microcapsule containing a multifunctional methylhydrosiloxane-dimethylsiloxane copolymer crosslinker. Following this, the reactivity of the PMMA/crosslinker microcapsule is evaluated in the presence of a vinyl-terminated PDMS at $50{ }^{\circ} \mathrm{C}$ and $120{ }^{\circ} \mathrm{C}$, respectively. To characterise the system quantitatively, time sweep rheological analyses are used, which determine the storage modulus of the mixture containing the PMMA/crosslinker microcapsule and the vinyl-terminated PDMS. In the last part of the paper, mixtures with different stoichiometric imbalances are characterised through time sweep rheological measurements, and the rheological properties of the obtained network are compared with those of a pure PDMS elastomer.

\section{Experimental section}

\section{Materials}

The chemicals employed are: poly (methyl methacrylate) (PMMA) $\left(M_{\mathrm{w}}=15000 \mathrm{~g} \mathrm{~mol}^{-1}\right.$, Aldrich), 25-35\% (methylhydrosiloxane) with $65-70 \%$ (dimethylsiloxane) copolymer (HMS-301) $\left(M_{\mathrm{n}}=2000 \mathrm{~g} \mathrm{~mol}{ }^{-1}\right.$, 8-functional crosslinker, Gelest), platinum cyclovinylmethysiloxane complex (SIP 6832.2 catalyst, Gelest), vinyl-terminated polydimethylsiloxane (DMS-V35) $\left(M_{\mathrm{n}}=49500 \mathrm{~g} \mathrm{~mol}^{-1}\right.$, Gelest), chloroform (>99\%, Aldrich), heptane (>99\%, Aldrich), methanol (>99\%, Aldrich) and deuterated chloroform ( $>99.8 \% \mathrm{D}$, Aldrich).

\section{Apparatus}

An air compressor (\#1A) and an airbrush (DH-201) were purchased from Sparmax (Germany). The compressor's air flow ranged from 7 to 11 litres per minute, and the maximum pressure provided by the compressor in the air compressing process was $40 \mathrm{psi}$. The diameter of the nozzle in the airbrush was $0.8 \mathrm{~mm}$.

The morphology of the PMMA/HMS-301 microcapsules was analysed with a scanning electron microscope (SEM) FEI Inspect $\mathrm{S}$, Oxford Instruments, with an acceleration voltage of $10-20 \mathrm{kV}$.

The size distribution of the PMMA/HMS-301 microcapsules was measured by Mastersizer (Malvern, UK) in a jar tester. The instrument was equipped with a laser at a wavelength of $633 \mathrm{~nm}$ and a size range of $1 \mu \mathrm{m}-1 \mathrm{~mm}$.

${ }^{1} \mathrm{H}$ NMR spectra were obtained on a Bruker $250 \mathrm{MHz}$ NMR spectrometer in $\mathrm{CDCl}_{3}$ at room temperature. ${ }^{1} \mathrm{H}$ chemical shifts were referenced to TMS via a residual non-deuterated solvent signal at $\delta=7.26 \mathrm{ppm}$.

Rheological measurements were performed in an AR2000 stress-controlled rheometer, while measurements were taken with a strain of $2 \%$ to ensure they were within the linear regime of the material ${ }^{17}$ as well as to minimise any disruption to the network. Similar to other measurements of in situ crosslinking reactions of additional curing silicones, ${ }^{\mathbf{1 8 , 1 9}}$ the applied frequency was set to $1 \mathrm{~Hz}$.

\section{Microcapsule preparation}

The polymeric solution was prepared by dissolving $1.0 \mathrm{~g}$ of HMS-301 and $1.0 \mathrm{~g}$ of PMMA in $5.4 \mathrm{~mL}$ chloroform. The polymeric solution was stirred at $700 \mathrm{rpm}$ with a magnetic stirrer for 24 hours at room temperature. Next, the polymeric solution was sprayed with an airbrush into a beaker containing $200 \mathrm{~mL}$ methanol. The micro droplets formed from the polymeric solution precipitated, resulting in the encapsulation of HMS301. The microcapsules were then washed with methanol several times, in order to remove any residual HMS-301 on the surface. Finally, the microcapsules were collected by filtration. Fig. 1 shows the scheme for the spraying setup.

\section{Microcapsule preparation with different HMS-301 concentrations}

In order to compare the encapsulation efficiency of the microcapsules, different concentrations of HMS-301 were used in the preparation as follows: (a) 10\% (wt) of HMS-301 and 10\% (wt) of PMMA in $80 \%$ (wt) chloroform (MP10H10C), (b) 15\% (wt) of HMS-301 and 10\% (wt) of PMMA in 75\% (wt) chloroform (MP10H15C) and (c) 20\% (wt) of HMS-301 and 10\% (wt) of PMMA in 70\% (wt) chloroform (MP10H20C).

For comparison purposes, microcapsules without HMS-301 were prepared using the same procedure from a polymeric solution containing 10\% (wt) of PMMA and 90\% (wt) of chloroform.

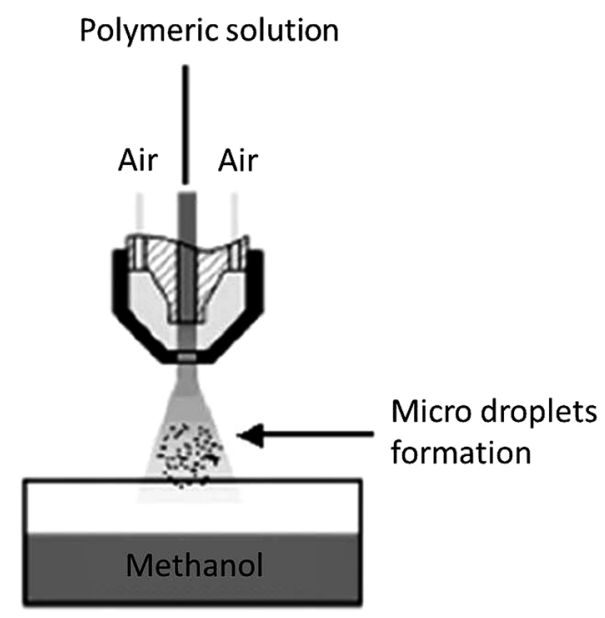

Fig. 1 Schematic diagram of the spraying setup. 


\section{Soluble fraction determination}

Approximately $0.5 \mathrm{~g}$ of network obtained from microcapsule (MP10H10C) + V35 as well as HMS-301 + V35 with different stoichiometric imbalance was swelled in heptane (20-30 times excess, i.e. 15-25 mL solvent to a sample of $0.5 \mathrm{~g}$; in all experiments, we ensure that the networks were fully covered in heptane) for $48 \mathrm{~h}$. After the $48 \mathrm{~h}$, the networks were separated from the heptane solution. The residual heptane was removed by evaporation for $48 \mathrm{~h}$ under atmospheric condition.

\section{Results and discussion}

\section{Morphological characterisation}

Fig. 2a and b show the representative SEM graphs of the outer surfaces and a cross-sectional view of the PMMA/HMS-301 microcapsules which were washed with methanol and heptane, respectively. Fig. 2a shows that the microcapsules were spherically shaped and only a few were broken after washing with methanol. Due to the low solubility of HMS-301 in methanol, methanol removed any HMS-301 on the surface only, without washing away HMS-301 inside the microcapsules. All the microcapsules in later sections were washed with methanol unless otherwise stated. Fig. $2 \mathrm{~b}$ shows that the microcapsules had a core-shell structure with a porous shell. Because of the high solubility of HMS-301 in heptane, heptane passed through the pores in the PMMA shell and entered into the core of the microcapsule. Thus, heptane removed most HMS-301 and destroyed some of microcapsules during evaporation, thereby leaving a hollow structure in the microcapsules.

The morphology of the PMMA shell was similar to that of the polysulfone (PSU) shell of the PSU/vanillin microcapsule. ${ }^{20}$ Similar to our preparation process, the PSU/vanillin microcapsule was also prepared by spraying polymeric solution containing PSU and vanillin into a non-solvent by using an air brush..$^{2021}$ As well as studies on PSU/vanillin microcapsules, several others have proven that microcapsules with a porous shell are suitable for stimuli-responsive controlled release, in order to obtain fast response times..$^{20,22,23}$ Consequently, PMMA/ HMS-301 microcapsules should have a potential for controlled release when stimulated.
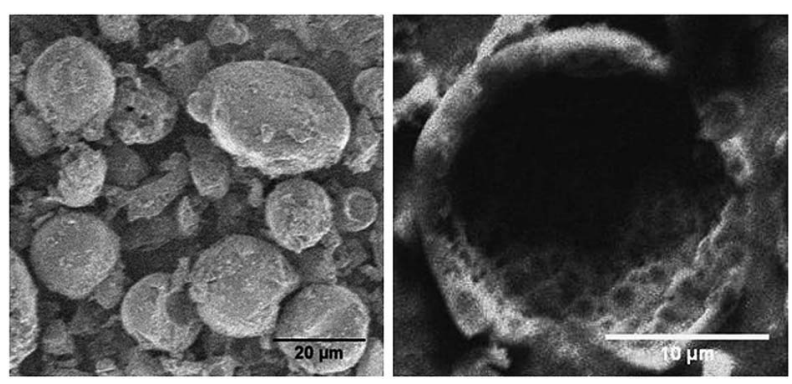

Fig. 2 (a (left) and b (right)). SEM image of the outer surface (left) and a cross-section (right) of PMMA/HMS-301 microcapsules. The left- and the right-hand SEM images show the morphology of the MP1OH1OC microcapsules washed with methanol and heptane, respectively.
Size distribution of PMMA/HMS-301 microcapsules and empty PMMA capsules

As discussed in the previous section, most of the microcapsules were spherical after washing with methanol, and they should also have been identical to those not washed with methanol, as methanol only removed HMS-301 on the surface. The size distribution of the microcapsules could therefore be investigated with Mastersizer, based on laser diffraction. Fig. 3 shows the size distribution of the microcapsules with a crosslinker inside as well the empty PMMA capsules. As shown in Fig. 3, microspheres had a mean diameter ranging from $47 \mu \mathrm{m}$ to 58 $\mu \mathrm{m}$ and were monomodal and similar in size distribution.

\section{Determination of the HMS-301 weight fraction in microcapsules}

The weight fraction of HMS-301 was determined through ${ }^{1} \mathrm{H}$ NMR spectroscopy. As the $-\mathrm{O}-\mathrm{CH}_{3}$ groups in PMMA and the $\mathrm{Si}-\mathrm{H}$ groups in HMS-301 have ${ }^{1} \mathrm{H}$ chemical shifts of $3.6 \mathrm{ppm}$ and $4.7 \mathrm{ppm}$, respectively, the mol ratio between the PMMA and HMS-301 could be calculated from the corresponding area of the resonances. Fig. 4 shows the integrated signals of the ${ }^{-} \mathrm{O}-\mathrm{CH}_{3}$ groups and the $\mathrm{Si}-\mathrm{H}$ groups in the ${ }^{1} \mathrm{H}$ NMR spectra. The weight fractions of HMS-301, calculated by ${ }^{1} \mathrm{H}$ NMR, were $28 \%$, $21 \%$ and $14 \%$ in MP10H10C, MP10H15C and MP10H20C, respectively. For more details on the method used in determining the content of HMS-301, see ESI. $\dagger$

Both the mean diameter and the weight fraction of the HMS301 of PMMA/HMS-301 microcapsules are summarized and shown in Table 1.

With the increased HMS-301 concentration, the viscosity of the solution decreased, resulting in a delayed breakup process and impeded atomization. Thus the encapsulation efficiency was shown to decrease upon decrease of the shell material concentration.

The size distribution and mean diameter depend on several factors such as the viscosity and surface tension of the solution being atomized as well as the turbulence created. ${ }^{24}$ The

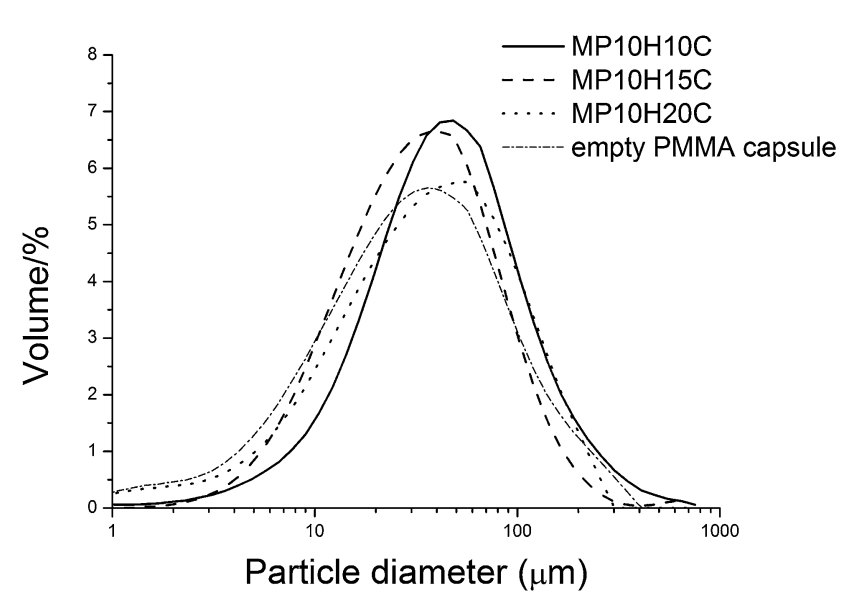

Fig. 3 Size distribution of MP10H10C, MP10H15C, MP10H20C microcapsules and empty PMMA capsules. 


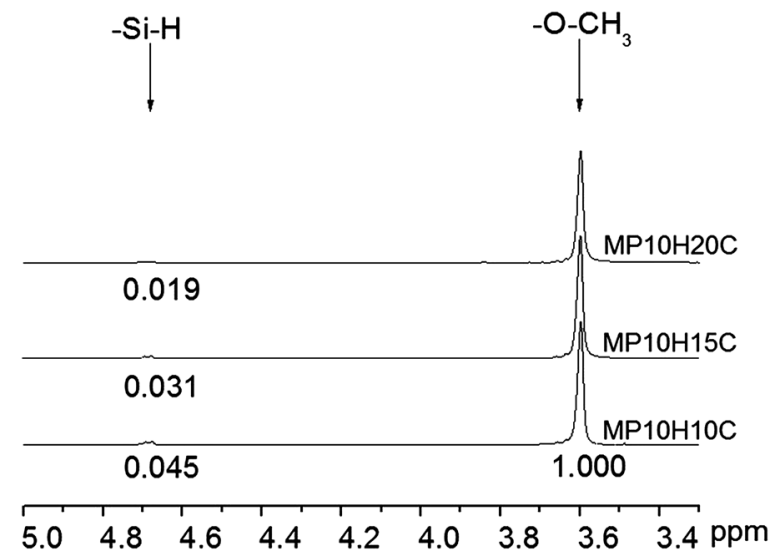

Fig. $4{ }^{1} \mathrm{H}$ NMR spectra of MP10H10C, MP10H15C and MP10H20C microcapsules with an indication of the characteristic peaks for $\mathrm{Si}-\mathrm{H}$ and $-\mathrm{O}-\mathrm{CH}_{3}$.

deviation in the mean diameters is similar to what is observed for other similar systems. ${ }^{25}$

\section{Reactivity of PMMA/HMS-301 microcapsules in a vinyl- terminated PDMS polymer}

To evaluate the reactivity of the microcapsule in a melt of vinylterminated PDMS, a typical mixture containing a $0.01 \mathrm{~g}$ PMMA/ HMS-301 microcapsule (MP10H10C) and $1 \mathrm{~g}$ V35 was made. All mixtures in the experiments contained 7 ppm platinum catalyst. Identical mixtures were placed in two vials, which were then inserted into ovens at $50{ }^{\circ} \mathrm{C}$ and $120{ }^{\circ} \mathrm{C}$ and cured for several days. Fig. 5a shows that the mixture cured at $50{ }^{\circ} \mathrm{C}$ was translucent and viscous, with no visible settling of PDMS gel. The mixture remained viscous and of low viscosity, as the HMS-301 remained sequestered inside the rigid PMMA shell, leaving V35 unable to react. In contrast, Fig. 5b shows that a crosslinked gel was obtained when the mixture was heated to $120{ }^{\circ} \mathrm{C}$. This indicated an extensive crosslinking reaction between HMS-301 and V35. After cooling down to room temperature, the material remained in its gel state, indicating that the gelation did not arise from any physical association between the PMMA/HMS301 microcapsule and V35.

To characterise the rheological behaviour of the PMMA/ HMS-301 microcapsule and V35 mixture quantitatively, time sweep rheological measurements were performed at $50{ }^{\circ} \mathrm{C}$ and $120{ }^{\circ} \mathrm{C}$. The measurements were performed with a low amplitude strain, in order to minimise any disruption to the network
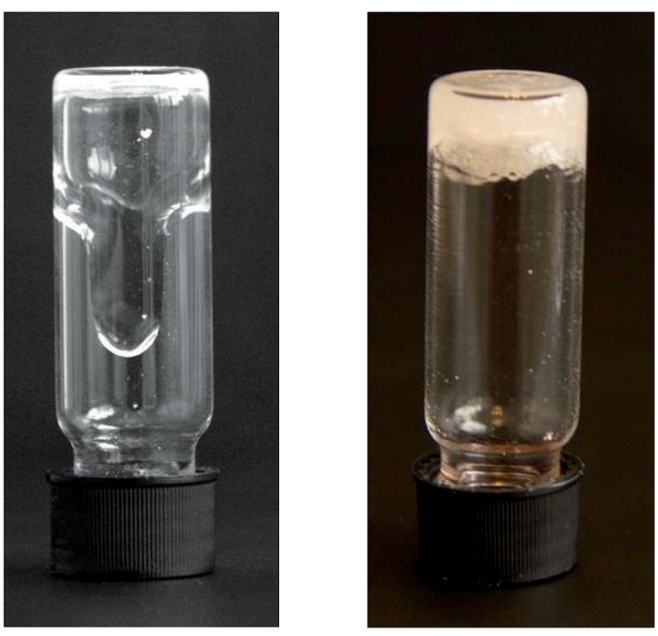

Fig. 5 (a (left), 5b (right)). Photograph of mixtures containing the PMMA/HMS-301 microcapsule and V35 after curing at $50{ }^{\circ} \mathrm{C}$ (left) and $120{ }^{\circ} \mathrm{C}$ (right).

during the formation process. Table 2 shows the composition and stoichiometric imbalance of the mixture in the measurements. The stoichiometric imbalance $(r)$ is the ratio between the mol number of the hydride groups and the vinyl groups. $r$ is calculated from:

$$
r=\frac{n_{\text {hydride }}}{n_{\text {vinyl }}}=\frac{\frac{m_{\mathrm{HMS}-301}}{M_{\mathrm{HMS}-301}} f_{\mathrm{HMS}-301}}{\frac{m_{\mathrm{V} 35}}{M_{\mathrm{V} 35}} f_{\mathrm{V} 35}}
$$

where $n$ is the number of moles, $m$ is the mass, $M$ is the molecular weight and $f$ is the functionality of the respective molecule, and where subscripts HMS-301 and V35 denote the hydride crosslinker and vinyl-terminated PDMS, respectively.

Fig. 6 shows representative curves of the storage modulus of the mixture containing the PMMA/HMS-301 microcapsule and

Table 2 Compositions and stoichiometric imbalance in rheological measurements

\begin{tabular}{llll}
\hline$m_{\mathrm{V} 35} / \mathrm{g}$ & Sample & $m_{\text {microcapsule }} / \mathrm{g}$ & $\begin{array}{l}\text { Stoichiometric } \\
\text { imbalance }(r)\end{array}$ \\
\hline 1.013 & MP10H10C & 0.010 & 0.24 \\
0.992 & MP10H15C & 0.013 & 0.24 \\
1.005 & MP10H20C & 0.020 & 0.24
\end{tabular}

Table 1 Mean diameter and weight fraction of HMS-301 for the PMMA/HMS-301 microcapsules

\begin{tabular}{|c|c|c|c|c|c|}
\hline \multirow[b]{2}{*}{ Sample ID } & \multicolumn{3}{|c|}{ Concentration in solution before spray (wt\%) } & \multirow{2}{*}{$\begin{array}{l}\text { Weight fraction } \\
\text { of HMS-301 (wt\%) }\end{array}$} & \multirow[b]{2}{*}{ Mean diameter $(\mu \mathrm{m})$} \\
\hline & PMMA & HMS-301 & Chloroform & & \\
\hline MP10H10C & 10 & 10 & 80 & 28 & 48 \\
\hline MP10H15C & 10 & 15 & 75 & 21 & 41 \\
\hline MP10H20C & 10 & 20 & 70 & 14 & 56 \\
\hline Empty PMMA capsules & 10 & 0 & 90 & 0 & 41 \\
\hline
\end{tabular}




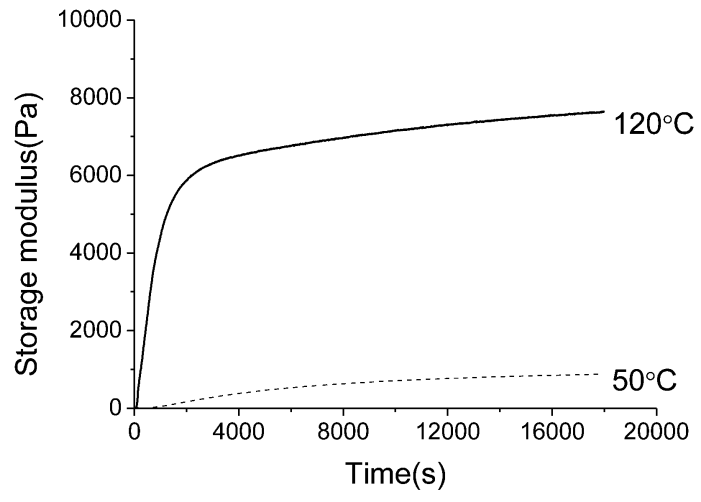

Fig. 6 Development of elasticity in mixtures containing the PMMA/ HMS-301 microcapsule (MP10H10C) and V35 at $50{ }^{\circ} \mathrm{C}$ (dash) and $120^{\circ} \mathrm{C}$ (solid) over a period of 5 hours.

V35 at $50{ }^{\circ} \mathrm{C}$ and $120{ }^{\circ} \mathrm{C}$, respectively. At $50{ }^{\circ} \mathrm{C}$, the storage modulus remained low $(\sim 500 \mathrm{~Pa})$ and was lower than the loss modulus (not shown in the figure), thereby indicating that the mixture maintained viscous behaviour. On the other hand, a slight increase in the storage modulus of the mixture suggested that there was a small degree of crosslinking reaction between HMS-301 and V35, which was related to the leakage of a small amount of HMS-301 from the porous PMMA shell. The porosity of the PMMA shell can be seen in the SEM image in Fig. $2 \mathrm{~b}$. When measurements were made at $120{ }^{\circ} \mathrm{C}$, the storage modulus of the mixture increased rapidly, eventually reaching a plateau within 5 hours $(\sim 8000 \mathrm{~Pa})$, after which any further increase in storage modulus was minimal. $80 \%$ of the maximal elasticity (determined from the curve) was obtained within the first 30 minutes. This massive increase in the storage modulus of the mixture not only suggested that there was a substantial degree of crosslinking reaction during the measurement, but also corresponded well with our visual observation of the crosslinked gel obtained in the vial at $120{ }^{\circ} \mathrm{C}$ (shown in Fig. 5b).

Considering the different rheological behaviours of the mixture at $50{ }^{\circ} \mathrm{C}$ and $120{ }^{\circ} \mathrm{C}$, the results indicated that the reactivity of the PMMA/HMS-301 microcapsule at $120{ }^{\circ} \mathrm{C}$ was significantly higher that at $50{ }^{\circ} \mathrm{C}$. This difference in reactivity could be explained as follows: at $50{ }^{\circ} \mathrm{C}$, most of the HMS-301 was sequestered in the PMMA shell, meaning the mixture was unable to react. Moving towards the situation at $120{ }^{\circ} \mathrm{C}$, the temperature was higher than the $T_{\mathrm{g}}$ of the PMMA $\left(T_{\mathrm{g}}=100^{\circ} \mathrm{C}\right),{ }^{13}$ resulting in the softening of the PMMA shell in the microcapsule and the release of HMS-301. Consequently, this release of HMS-301 reacted with V35 in the presence of a catalyst, yielding a PDMS network.

\section{Soluble fraction}

To evaluate the soluble fraction of the network obtained from the MP10H10C + V35 with different stoichiometric imbalances, swelling experiments were performed. In this and the following section, MP10H10C was selected as a representative microcapsule, as the HMS-301 content was the highest among all the samples. In order to interpret the soluble fraction of the network from MP10H10C + V35, reference networks with identical stoichiometric imbalance as the MP10H10C + V35 network were prepared by mixing HMS-301 + V35 and cured under identical conditions. Table 3 shows that the soluble fraction of the network from MP10H10C + V35 ranged from $14.5 \%$ to $27.8 \%$ and that of reference samples ranged from $5.6 \%$ to $18.2 \%$. The soluble fraction in networks can be attributed to inactive species within the reactants and imperfections arising during the crosslinking of the network. Since the inactive species were identical for all samples, the difference in soluble fraction between the MP10H10C + V35 network and referenced network arises from different degrees of imperfections in the networks. ${ }^{13}$ The PMMA is not distinguishable soluble in heptane and is thus not washed out of the networks. The difference in the imperfections in the network could be explained as follows: Upon the heating of the mixture containing MP10H10C and V35, the HMS-301 diffused out from the microcapsule and reacted with V35, yielding crosslinked PDMS network around the microcapsule. The locally formed network has a much higher viscosity than the vinyl terminated PDMS, eventually impeding the diffusion of HMS-301 and the crosslinking reaction. As a result, the unreacted PDMS remained in the network and increased the imperfections as well as the soluble fraction of the MP10H10C + V35 network.

It was further found that the soluble fraction of the MP10H10C + V35 network decreased from $27.8 \%$ to $14.5 \%$ when the stoichiometric imbalance increased from 0.8 to 1.4. This trend was similar to that of the referenced system, indicating that the increasing amount of microcapsule resulted in the increasing released amount of HMS-301 and yielding stronger network with lower soluble fraction. ${ }^{\mathbf{1 3 , 1 4}}$

\section{Influence of stoichiometric imbalance on the equilibrium storage modulus}

The aim of this section is to investigate the influence of stoichiometric imbalance on the equilibrium storage modulus of the network obtained from the crosslinking reaction between the PMMA/HMS-301 microcapsule and V35. Mixtures containing the PMMA/HMS-301 microcapsule and V35 (System A) were characterised by a time sweep rheological measurement at $120{ }^{\circ} \mathrm{C}$ within the range $0.2<r<1.4$. In order to interpret the rheological properties of the networks in System A, HMS-301 + empty PMMA capsule + V35 (System B) and HMS-301 + V35 (System C) with $0.6<r<1.4$ were prepared and then characterised by a time sweep rheological measurement at $120{ }^{\circ} \mathrm{C}$. System B was made to simulate the situation whereby all HMS301 diffused out from the PMMA shell and mixed with V35, while System C consisted of a PDMS elastomer without any filler and was used as a reference sample (Table 4).

The rheological behaviours of Systems A, B and C in a time sweep rheological measurement are shown in ESI S2. $\dagger$ The equilibrium storage moduli of Systems A, B and C were obtained from Fig. S2 in ESI S2 $\uparrow$ and are shown in Fig. 7, where we can see that the equilibrium storage modulus of System A increased in line with an increase in stoichiometric imbalance in the range $0.2<r<0.8$, indicating that the crosslinking density 
Table 3 Soluble fraction of networks obtained from MP10H10C + V35 and HMS301 + V35

\begin{tabular}{|c|c|c|c|c|}
\hline & \multicolumn{4}{|c|}{$\begin{array}{l}\text { Stoichiometric } \\
\text { imbalance }(r)\end{array}$} \\
\hline & 0.8 & 1 & 1.2 & 1.4 \\
\hline $\begin{array}{l}\text { Soluble fraction of network } \\
\text { obtained from MP10H10C + V35 (\%) }\end{array}$ & 27.8 & 21.5 & 20.3 & 14.5 \\
\hline $\begin{array}{l}\text { Soluble fraction of network obtained from } \\
\text { reference system (HMS-301 + V35) (\%) }\end{array}$ & 18.2 & 10.9 & 8.4 & 5.6 \\
\hline
\end{tabular}

of the network increased as more PMMA/HMS-301 microcapsules were used in this range. However, in the range $0.8<r<1.4$, the equilibrium storage modulus of System A remained almost constant. This suggested that the crosslinking density of the network did not increase when an excess amount of PMMA/ HMS-301 microcapsules was used in the mixture. In comparison, the maximum equilibrium storage moduli of most commonly applied PDMS networks are obtained in the range $1.2<r<1.4,{ }^{15}$ whereas the maximum equilibrium storage modulus of System A was obtained at $r=0.8$. This indicated that HMS-301 was trapped in the range $0.8<r<1.4$, due to diffusion constriction. The reason for this trapping of HMS-301 is explained in the following: upon heating the mixture containing the PMMA/HMS-301 microcapsule and V35, the PMMA shell softened, thus resulting in the release of HMS-301. Then, the released HMS-301 reacted with V35 around the microcapsule, yielding locally a crosslinked PDMS network. The crosslinked PDMS network around the microcapsule had a much higher viscosity than V35, such that the further diffusion of HMS-301 was significantly hindered by the crosslinked PDMS network. This phenomenon was described by Ndoni and Kramer as 'strangulation within polymer networks'. ${ }^{26}$ As the crosslinked PDMS network accumulated around the microcapsule, the diffusion of HMS-301 would eventually stop, resulting in the trapping of HMS-301.

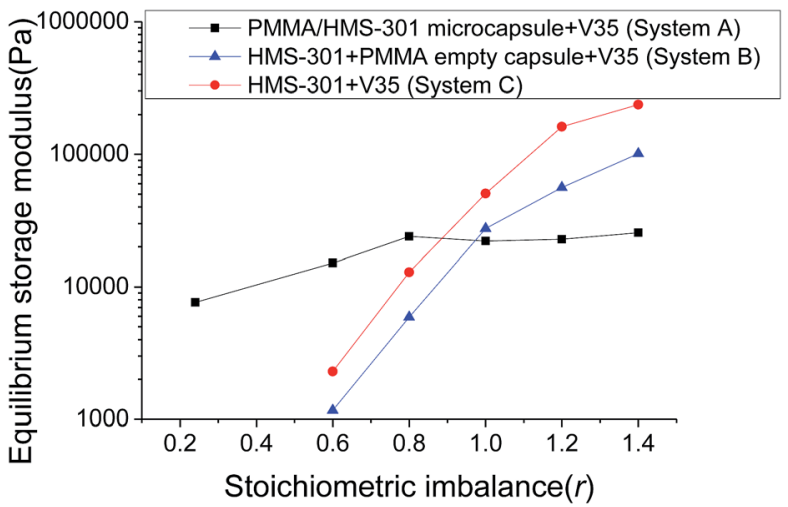

Fig. 7 Equilibrium storage modulus of the resulting networks from PMMA/HMS-301 microcapsule (MP10H10C) +V35, HMS-301 + empty PMMA capsule + V35 and HMS-301 + V35 as a function of $r$.

The results of System B and System C clearly showed that the equilibrium storage modulus increased as stoichiometric imbalance increased in the interval $0.6<r<1.4$. For most of the PDMS network, the maximum equilibrium storage modulus was obtained in the range $1.2<r<1.4$, which was in line with our experimental results. In the comparison of System B and System C, the equilibrium storage modulus of System B was lower than that of System $\mathrm{C}$ at identical stoichiometric imbalance. This could be attributed to the immiscibility between the PDMS and the empty PMMA capsule. As such, this immiscibility increased the heterogeneity of the PDMS network, thereby causing the network's lower equilibrium storage modulus.

When comparing the rheological properties of Systems A, B and $\mathrm{C}$, the equilibrium storage modulus of System A was higher than that of Systems B and $\mathrm{C}$ in the range $0.2<r<0.8$. The high storage modulus of System A in this range could be attributed to the reinforcing effect of the PMMA shell from the PMMA/HMS301 microcapsule. ${ }^{27}$ The reinforcing effect is explained as follows: when the mixture containing the PMMA/HMS-301 microcapsule and V35 was heated up to $120{ }^{\circ} \mathrm{C}, \mathrm{HMS}-301$

Table 4 Composition and stoichiometric imbalance of Systems A, B and C

\begin{tabular}{|c|c|c|c|c|}
\hline System ID & & $r$ & $m_{\text {(PMMA/HMS-301 microcapsule) }} / g$ & $m_{\text {(PMMA empty capsules) }} / \mathrm{g}$ \\
\hline \multirow[t]{6}{*}{ A } & PMMA/HMS-301 microcapsule + V35 & 0.2 & 0.010 & - \\
\hline & & 0.6 & 0.027 & - \\
\hline & & 0.8 & 0.033 & - \\
\hline & & 1.0 & 0.045 & - \\
\hline & & 1.2 & 0.058 & - \\
\hline & & 1.4 & 0.067 & - \\
\hline \multirow[t]{5}{*}{ B } & HMS-301 + empty PMMA capsules + V35 & 0.6 & - & 0.018 \\
\hline & & 0.8 & - & 0.023 \\
\hline & & 1.0 & - & 0.034 \\
\hline & & 1.2 & - & 0.042 \\
\hline & & 1.4 & - & 0.045 \\
\hline \multirow[t]{5}{*}{$\mathrm{C}$} & HMS-301 + V35 & 0.6 & - & - \\
\hline & & 0.8 & - & - \\
\hline & & 1.0 & - & - \\
\hline & & 1.2 & - & - \\
\hline & & 1.4 & - & - \\
\hline
\end{tabular}




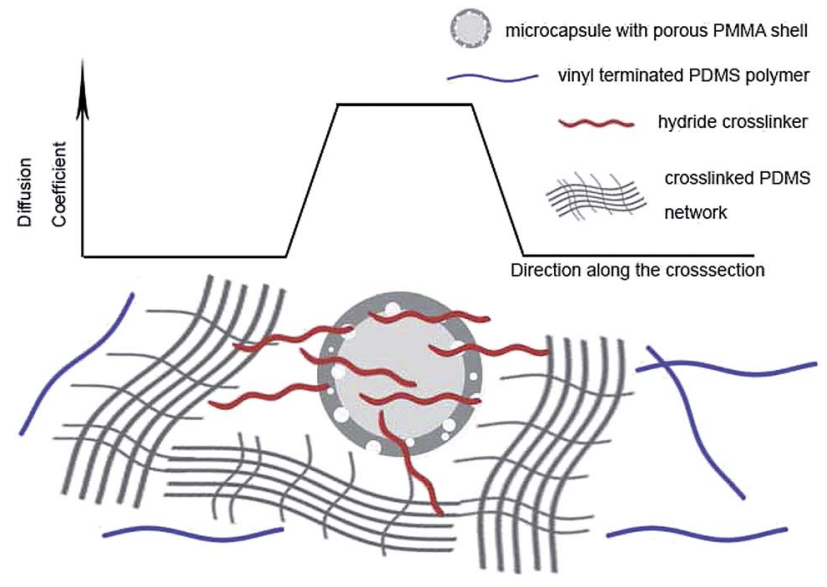

Fig. 8 Schematic diagram of the formed PDMS network around the microcapsule that leads to the trapping of HMS-301 in the range $0.8<r<1.4$

diffused out from the PMMA shell. The released HMS-301 reacted with V35 around the microcapsule, yielding crosslinking points around the microcapsules. Locally formed PDMS gel adhered to the porous PMMA shell, eventually anchoring the PDMS chain to the porous PMMA shell, which consequently reinforced the network and increased its storage modulus. ${ }^{28}$

In the range $1.0<r<1.4$, the equilibrium storage modulus of Systems B and C was significantly higher than that of System A with an identical stoichiometric imbalance. This indicated that the equilibrium storage modulus was determined by the homogeneity of the network in this range. The homogeneity of the network of System A was constrained by the trapping of HMS-301, as explained in the previous discussion. The trapping of HMS-301 - caused by the PDMS network around the microcapsule - is envisioned in Fig. 8.

Despite the low storage moduli of the obtained PDMS elastomer $(\sim 30 \mathrm{kPa})$, it is comparable to that of so-called heterogeneous bimodal networks developed by Bejenariu et al. (10$300 \mathrm{kPa})^{17}$ as well as Madsen et al. $(10-100 \mathrm{kPa}){ }^{29}$ To increase the storage moduli of the network, a natural extension of this study would be to fill the liquid silicone (V35) with fumed silica to reinforce the resulting elastomer. This will increase the storage modulus dramatically and cause elastic moduli comparable to that of commercial silicone elastomer formulations. However, current materials for fracture sealing include hydrogels with significantly lower elastic moduli ${ }^{30-33}$ so for that particular application no further filler reinforcement is deemed necessary.

\section{Conclusions}

Control of the crosslinking reaction between a vinyl-terminated PDMS and a hydride crosslinker has been achieved by encapsulating a HMS-301 hydride crosslinker in a PMMA shell. This PMMA/HMS-301 microcapsule was mixed with a V35 vinylterminated PDMS to create a stable mixture which remained viscous at $50{ }^{\circ} \mathrm{C}$, thereby indicating that the mixture did not react and that the HMS-301 was sequestered in the PMMA shell. In contrast, the mixture formed a gel when heated up to $120^{\circ} \mathrm{C}$, suggesting that an extensive crosslinking reaction took place between HMS-301 and V35, due to the release of HMS-301 from the microcapsule. As the amount of HMS-301 released determined the crosslinking density and the storage modulus of the PDMS network, the influence of stoichiometric imbalance on the equilibrium storage modulus of the PDMS network was investigated. It was found that the addition amount of a microcapsule increased the equilibrium storage modulus of the PDMS network in the range $0.2<r<0.8$. It was also found that the equilibrium storage modulus of the PDMS network remained constant in the range $0.8<r<1.4$, due to the stalled diffusion of the HMS-301 by the locally formed PDMS network around the microcapsule. This indicated that the equilibrium storage modulus of the PDMS network became less sensitive towards stoichiometric imbalance in the control crosslinking reaction.

In applications where high modulus and transparency are not required, soft PDMS elastomer obtained from controlled crosslinking reaction can be utilized, e.g. as elastomeric plugs in oil fields. Furthermore, the optimum amount of microcapsules used in the controlled crosslinking reaction was found in the present study, which allows for designing an optimised controlled crosslinking system.

\section{References}

1 A. P. R. Johnston, G. K. Such and F. Caruso, Angew. Chem., Int. Ed. Engl., 2010, 49, 2664-2666.

2 B. J. Blaiszik, S. L. B. Kramer, S. C. Olugebefola, J. S. Moore, N. R. Sottos and S. R. White, Annu. Rev. Mater. Res., 2010, 40, 179-211.

3 L. Gonzalez, B. Ma, K. Malgorzata, L. Li, H. J. Hansen, S. Hvilsted and A. L. Skov, Macromol. Mater. Eng., 2014, DOI: 10.1002/mame.201400020.

4 Y. Deyrail, N. Zydowicz and P. Cassagnau, Polymer, 2004, 45, 6123-6131.

5 A. P. Esser-Kahn, S. A. Odom, N. R. Sottos, S. R. White and J. S. Moore, Macromolecules, 2011, 44, 5539-5553.

6 S. H. Cho, S. R. White and P. V. Braun, Adv. Mater., 2009, 21, 645-649.

7 C. L. Mangun, C. Mader, N. R. Sottos and S. R. White, Polymer, 2010, 51, 4063-4068.

8 J. E. Mark and J. L. Sullivan, J. Chem. Phys., 1977, 66, 10061011.

9 K. Urayama, T. Kawamura and S. Kohjiya, J. Chem. Phys., 1996, 105, 4833-4840.

10 S. Patel, S. Malone and C. Cohen, Macromolecules, 1992, 25, 5241-5251.

11 F. Chambon and H. H. Winter, J. Rheol., 1987, 31, 683-697. 12 H. H. Winter and F. Chamson, J. Rheol., 1986, 30, 367-382.

13 S. M. G. Frankær, M. K. Jensen, A. G. Bejenariu and A. L. Skov, Rheol. Acta, 2012, 51, 559-567.

14 M. Villar, M. Bibbo and E. Valles, Macromolecules, 1996, 29, 4072-4080. 
15 A. L. Larsen, K. Hansen, O. Hassager, A. Bach, S. Ndoni and M. Jørgensen, Macromolecules, 2003, 36, 10063-10070.

16 D. Dasgupta, S. Manna and A. Garai, Macromolecules, 2008, 41, 779-787.

17 A. Bejenariu, L. Yu and A. Skov, Soft Matter, 2012, 8, 39173923.

18 T. J. Sanborn, P. B. Messersmith and A. E. Barron, Biomaterials, 2002, 23, 2703-2710.

19 E. Westhaus and P. B. Messersmith, Biomaterials, 2001, 22, 453-462.

20 B. Peña, C. Panisello, G. Aresté, R. Garcia-Valls and T. Gumí, Chem. Eng. J., 2012, 179, 394-403.

21 C. Panisello and R. Garcia-Valls, Ind. Eng. Chem. Res., 2012, 51, 15509-15516.

22 L. Chu, S. Park, T. Yamaguchi and S. Nakao, Langmuir, 2002, 18, 1856-1864.

23 L. Chu, S. Park, T. Yamaguchi and S. Nakao, J. Membr. Sci., 2001, 192, 27-39.
24 A. H. Lefebvre, Atomization and sprays, ed. N. Chigier, Hemisphere publishing corporation, West lafayette, US, 1989, pp. 14-25.

25 E. Herrero, E. Valle and M. Galan, Chem. Eng. J., 2006, 117, 137-142.

26 S. Ndoni and O. Kramer, Europhys. Lett., 1997, 39, 165-170.

27 M. I. Aranguren, E. Mora and J. V. DeGroot Jr, J. Rheol., 1992, 36, 1165-1182.

28 D. Ciprari, K. Jacob and R. Tannenbaum, Macromolecules, 2006, 39, 6565-6573.

29 F. B. Madsen, A. E. Daugaard, C. Fleury, S. Hvilsted and A. L. Skov, RSC Adv., 2014, 4, 6939.

30 G. A. Al-Muntasheri, I. A. Hussein, H. A. Nasr-El-Din and M. B. Amin, J. Pet. Sci. Eng., 2007, 55, 56-66.

31 M. Rafipoor, M. V. Sefti, F. Salimi, K. Jarrahian and S. S. Ghorashi, J. Dispersion Sci. Technol., 2013, 6, 1-31.

32 B. Sengupta, V. P. Sharma and G. Udayabhanu, J. Pet. Sci. Eng., 2012, 81, 145-150.

33 L. González, A. L. Skov and S. Hvilsted, J. Polym. Sci., Part A: Polym. Chem., 2013, 51, 1359-1371. 